\title{
Lipoatrophy and metabolic disturbance in mice with adipose-specific deletion of kindlin-2
}

\author{
Huanqing Gao,, Yuxi Guo, ${ }^{1}$ Qinnan Yan, ${ }^{1}$ Wei Yang, ${ }^{1}$ Ruxuan Li, ${ }^{1}$ Simin Lin, ${ }^{1}$ Xiaochun Bai, ${ }^{2}$ Chuanju \\ Liu, ${ }^{3}$ Di Chen, ${ }^{4}$ Huiling Cao, ${ }^{1}$ and Guozhi Xiao ${ }^{1,4}$ \\ ${ }^{1}$ Guangdong Provincial Key Laboratory of Cell Microenvironment and Disease Research, Shenzhen Key Laboratory \\ of Cell Microenvironment, and Department of Biology, Southern University of Science and Technology, Shenzhen, \\ China. 'Department of Cell Biology, School of Basic Medical Sciences, Southern Medical University, Guangzhou, China. \\ ${ }^{3}$ Department of Orthopedic Surgery and Department of Cell Biology, New York University School of Medicine, New York, \\ New York, USA. ${ }^{4}$ Department of Orthopedic Surgery, Rush University Medical Center, Chicago, Illinois, USA.
}

Kindlin-2 regulates integrin-mediated cell adhesion to and migration on the extracellular matrix. Our recent studies demonstrate important roles of kindlin- 2 in regulation of mesenchymal stem cell differentiation and skeletal development. In this study, we generated adipose tissue-specific conditional knockout of kindlin-2 in mice by using Adipoq-Cre BAC-transgenic mice. The results showed that deleting kindlin-2 expression in adipocytes in mice caused a severe lipodystrophy with drastically reduced adipose tissue mass. Kindlin-2 ablation elevated the blood levels of nonesterified fatty acids and triglycerides, resulting in massive fatty livers in the mutant mice fed with high-fat diet (HFD). Furthermore, HFD-fed mutant mice displayed type II diabetes-like phenotypes, including elevated levels of fasting blood glucose, glucose intolerance, and peripheral insulin resistance. Kindlin-2 loss dramatically reduced the expression levels of multiple key factors, including PPAR $\gamma$, mTOR, AKT, and $\beta$-catenin proteins, and suppressed adipocyte gene expression and differentiation. Finally, kindlin-2 loss drastically reduced leptin production and caused a high bone mass phenotype. Collectively, these studies establish a critical role of kindlin- 2 in control of adipogenesis and lipid metabolism as well as bone homeostasis.

Authorship note: $\mathrm{HG}, \mathrm{YG}$, and QY contributed equally to this work.

Conflict of interest: The authors have declared that no conflict of interest exists.

Copyright: (c) 2019, American Society for Clinical Investigation.

Submitted: February 26, 2019

Accepted: May 28, 2019

Published: July 11, 2019.

Reference information: /Cl Insight. 2019;4(13):e128405. https://doi. org/10.1172/jci.insight.128405.

\section{Introduction}

Adipose tissue plays a critical role in regulating energy balance and metabolism and its dysfunction is associated with metabolic diseases, such as obesity and insulin resistance. There are 2 main types of adipose in mammals, white adipose tissue (WAT) and brown adipose tissue (BAT). The former functions primarily in energy storage, while the latter dissipates energy through heat to maintain normal body temperature by the expression of uncoupling protein 1 (UCP1), which is located in the inner mitochondrial membrane and regulates proton reentry into the mitochondrial matrix to drive ATP synthesis (1)

Adipocytes produce hormones and adipokines that regulate food intake and nutrient homeostasis (2). Increased adiposity in obesity is closely correlated with increased risks for developing insulin resistance and diabetes mellitus, cardiovascular disease, and cancer (3-6). Adipose tissue expansion in obesity occurs through increased size of adipocytes loaded with excess fat or adipocyte hypertrophy, which is a characteristic of adipose tissue in obese individuals with metabolic syndrome. Increases in fat cell number (adipocyte hyperplasia) occur in obese individuals at the expense of large hypertrophic adipocytes.

Lipodystrophy, which is a lack of adipose tissue, associates with severe metabolic complications. For example, patients suffering from congenital generalized lipodystrophy usually develop insulin resistance, hypertriglyceridemia, and fatty liver $(7,8)$. Thus, maintaining proper adipose tissue mass and function is critical for normal metabolic homeostasis and understanding the underlying mechanisms has substantial clinical implications.

Kindlin-2, which is encoded by FERMT2, belongs to the kindlin protein family and is a component of cell-extracellular matrix adhesions. Kindlin-2 is expressed in multiple tissues and cell types, including mesenchymal stem cells, which can differentiate into osteoblasts, chondrocytes, and adipocytes (9). Kindlin-2 
binds to the $\beta$-integrin cytoplasmic tail and promotes integrin activation, which consequently mediates cell adhesion, spreading, and migration. Global deletion of kindlin-2 in mice results in peri-implantation lethality at E7.5, which demonstrates the critical role of kindlin-2 during early embryonic development. We utilized a conditional knockout strategy and demonstrated that kindlin-2 is essential for chondrogenesis by control of TGF- $\beta 1$ signaling and Sox9 expression during skeletal development (10). We further demonstrated that kindlin-2 plays a critical role in maintaining normal podocyte structure and function by association with the Rho GDP-dissociation inhibitor $\alpha$ (RhoGDI $\alpha$ ) and, thereby, suppresses Rac1 activation and protects podocytes from injury (11). More recently, we found that kindlin-2 controls bone morrow mesenchymal stem cell differentiation toward the osteoblastic or adipogenic lineage through control of YAP1/TAZ (12). Loss of kindlin-2 in these cells largely promoted adipogenic differentiation. However, it is not known whether kindlin-2 plays a direct role in regulation of adipocyte formation and differentiation during adipogenesis.

In this study, we utilized a conditional knockout strategy to selectively delete kindlin-2 expression in adipocytes and investigate the function of kindlin-2 in adipogenesis. We performed comprehensive analyses of mice in which kindlin-2 is ablated in adipocytes.

\section{Results}

Adipocyte-specific deletion of kindlin-2 drastically reduces adipose mass in mice. To investigate the role of kindlin-2 in adipogenesis, we deleted kindlin-2 expression in adipocytes by breeding floxed kindlin-2 (Kindlin-2 $2^{f / f}$ ) mice, in which exons 5 and 6 of the Kindlin-2 gene are flanked by 2 loxP sites (10), with Adipoq-Cre-transgenic mice harboring the Adipoq-Cre BAC transgene expressing Cre recombinase under the control of the mouse adiponectin (Adipoq) promoter/enhancer regions within the BAC transgene (13) and obtained Kindlin-2 $2^{f /+}$ Adipoq-Cre mice. We further bred the Kindlin-2 $2^{f /+}$ Adipoq-Cre mice with the Kindlin-2 $2^{f / f l}$ mice and generated

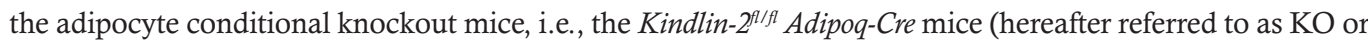
Kindlin-2-KO). The Kindlin-2-KO mice were viable, fertile, and born at the expected Mendelian frequency. Results from Western blotting revealed that the levels of kindlin-2 protein were markedly reduced in epididymal WAT (eWAT), inguinal WAT (iWAT), and BAT of KO mice, but not in non-adipose tissue, compared with those of WT mice (Figure 1, A and B). Quantitative RT-PCR analyses using RNAs from different adipose and non-adipose tissues revealed that the levels of Kindlin-2 mRNA were dramatically reduced in iWAT, eWAT, perirenal white adipose tissue (pWAT), and BAT of KO mice compared with those of control littermates (hereafter referred to as WT; Figure 1C). In contrast, levels of Kindlin-2 mRNA in non-adipose tissues, including kidney, liver, heart, and lung, were not significantly reduced in $\mathrm{KO}$ mice relative to those of WT control littermates (Figure 1D). Starting at 18 weeks of age, the KO mice fed with a normal chow diet (NCD) displayed a significant reduction in body weight compared with their control littermates (Figure 2, A and B). The mass of eWAT, iWAT, BAT, and pWAT was strikingly decreased in KO mice compared with that of WT mice (Figure 2, C-E). In contrast to results from adult mice, the values of eWAT, iWAT, and BAT were not significantly reduced in 3-week-old KO mice relative to WT control littermates (Supplemental Figure 1; supplemental material available online with this article; https://doi.org/10.1172/jci. insight.128405DS1). Notably, the weights of spleen and kidney normalized to the lengths of femurs were significantly increased in $\mathrm{KO}$ mice compared with those in WT mice (Figure 2, F and G).

Deleting kindlin-2 reduces adipocyte gene expression and differentiation. We next determined the expression levels of fat-related genes in KO mice by quantitative real-time RT-PCR analyses. The results showed that expression levels of adipocyte differentiation-dependent genes, including those encoding peroxisome proliferator-activated receptor gamma (Ppary), fatty acid binding protein 4 (Fabp4, also known as Ap2), and CCAAT/enhancer-binding protein alpha $(\operatorname{Cebp} \alpha)$, were all dramatically reduced in WAT, but not in BAT, of KO mice relative to those of WT mice (Figure 3, A and B). The transcript levels of the lipogenesis-related genes, including those encoding acetyl-CoA carboxylase (Acc), fatty acid synthase (Fas), and diacylglycerol O-acyltransferase 1 (Dgat1), were reduced in both WAT and BAT (Figure 3, A and B). The lipolysis-related genes, including those encoding hormone-sensitive lipase (Hsl) and adipose triglyceride lipase (Atgl), were reduced in WAT, but not BAT (Figure 3, A and B). The oxidation-related genes, including those encoding Ppara and acyl-CoA oxidase 1 (Acox1), were downregulated in WAT, but not in BAT (Figure 3, A and B). Histological analysis of adipose tissues of the 2 genotypes revealed that KO WAT adipocytes were smaller than WT WAT cells, which was consistent with BAT adipocytes (Figure 3, C-E). To determine whether the reduced WAT volume in $\mathrm{KO}$ mice was due to decreased size of adipocytes, we determined the relationship 
A

eWAT

wt1 wt2 ko1 ko2

$72 \mathrm{kD}$

$42 k D$

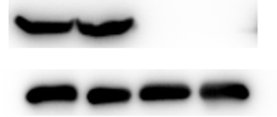

iWAT

wt1 wt2 ko1 ko2

$72 \mathrm{kD}$

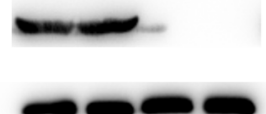

$42 k D$

\section{BAT}

wt1 wt2 ko1 ko2

$72 \mathrm{kD}$

$42 \mathrm{kD}$

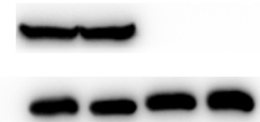

KINDLIN-2

ACTIN

KINDLIN-2

ACTIN
B

Brain Lung Heart Liver Spleen Kidney Muscle

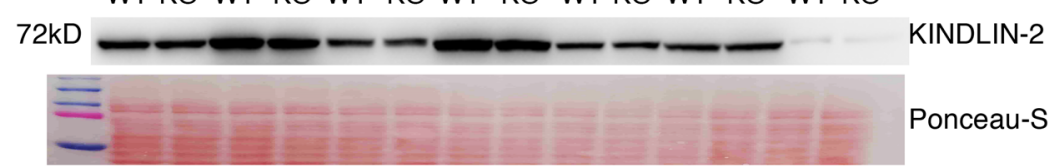

\section{C}

KINDLIN-2
ACTIN
KINDLIN-2
ACTIN

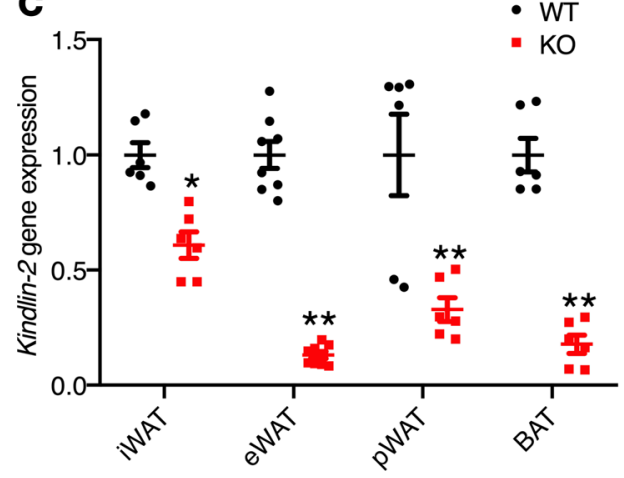

D

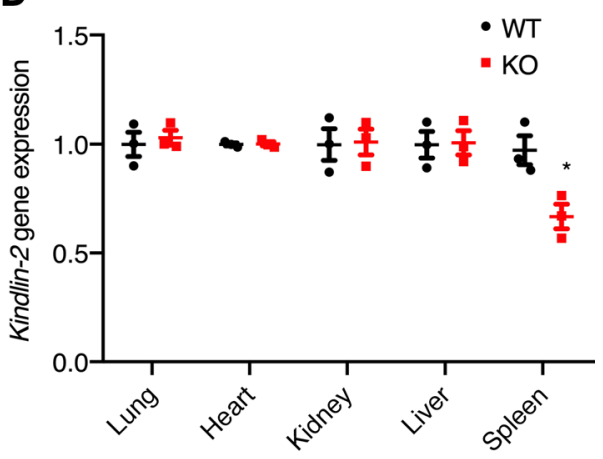

Figure 1. Adipose tissue-specific knockout of kindlin-2. (A) Western blot analysis of kindlin-2 expression in different adipose tissues. (B) Western blot analysis of kindlin-2 expression in non-adipose tissues. (C) Quantitative real-time reverse transcriptase PCR (qPCR) analyses of mRNA levels of kindlin-2 in different adipose tissues ( $N=6$ mice per group). (D) qPCR analyses of mRNA levels of kindlin- 2 in non-adipose tissues $\left(N=3\right.$ mice per group). ${ }^{*} P<0.05$, ${ }^{* *} P<0.01$ for KO vs. WT by Student's $t$ test.

between eWAT and adipocyte volumes. Analyzing the slope and intercept of the 2 genotypes showed that kindlin-2 loss reduced the size and number of adipocytes (Figure 3F).

Kindlin-2-KO mice fed with high-fat diet exhibit high fasting glucose levels, glucose intolerance, and peripheral insulin resistance. To investigate the role of kindlin-2 expression in adipocytes under pathological conditions, 6-week-old WT and KO mice were fed with a 60\% high-fat diet (HFD) for up to 18 weeks. Compared with the NCD-fed group, after 18 weeks on HFD, KO mice were less obese, weighing about $44 \mathrm{~g}$, which was $25 \%$ less than WT mice (Figure $4 \mathrm{~A}$ ). The size of eWAT, iWAT, and pWAT was dramatically smaller than that of their WT controls (Figure 4B). Quantitative analyses revealed that the weights of both WAT and BAT were significantly reduced in KO mice compared with those of WT mice (Figure 4, $\mathrm{C}$ and $\mathrm{D})$. The increases in weights of multiple organs, including liver, heart, lung, spleen, and kidney, were more significant in KO mice fed with HFD versus NCD (Figure 4, E and F). We next performed a glucose tolerance test (GTT) on WT and KO mice fed with HFD for 18 weeks. Results revealed that KO mice displayed an obvious glucose intolerance (Figure 4, G and H). After an acute glucose load, KO mice were less able to metabolize blood glucose, as indicated by the shift in the blood glucose curve in KO mice relative to WT mice; however, no difference in glucose tolerance was observed in WT and KO mice fed with NCD (Supplemental Figure 2, A and B). We further performed an insulin tolerance test (ITT) to determine whether this glucose intolerance observed in the KO mice fed with HFD was associated with peripheral insulin resistance. After 18 weeks on the HFD, the capacity of a bolus of insulin to reduce the blood glucose level was significantly reduced in $\mathrm{KO}$ mice only at the 120-minute time point, but not at the early time points $(30,60$, and 90 minutes; Figure 4 , I and J). In contrast, the capacity of insulin to reduce the blood glucose level was slightly, but significantly, increased in NCD-fed KO versus WT mice only at the 120-minute time point, but not at the early time points (30, 60, and 90 minutes; Supplemental Figure 2, C and D). The fasting blood glucose levels of KO mice were significantly higher than WT littermates (Figure 4K). Consistent with the dramatic lipoatrophy, serum levels of leptin and adiponectin were drastically reduced in KO mice relative to those of WT mice (Figure 4, L and M, and Supplemental Figure 2, E and F). Similar serum insulin levels were observed in KO and WT mice on HFD (Figure 4N). Notably, when fed with NCD, the KO mice exhibited significantly higher serum insulin levels than the 
A WT KO

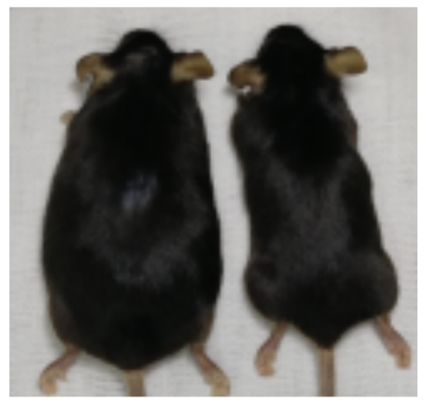

D

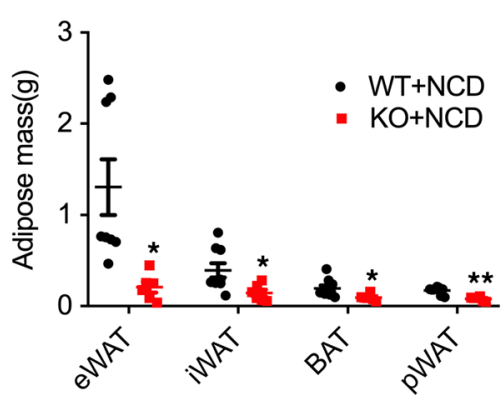

$\mathbf{F}$

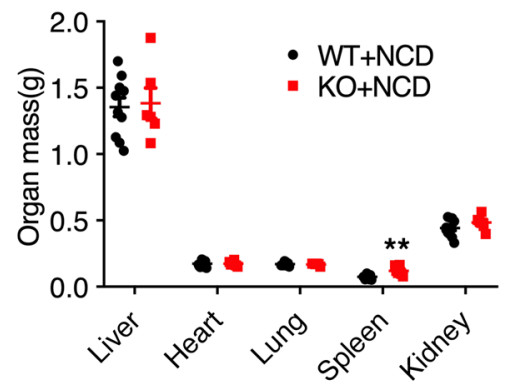

B

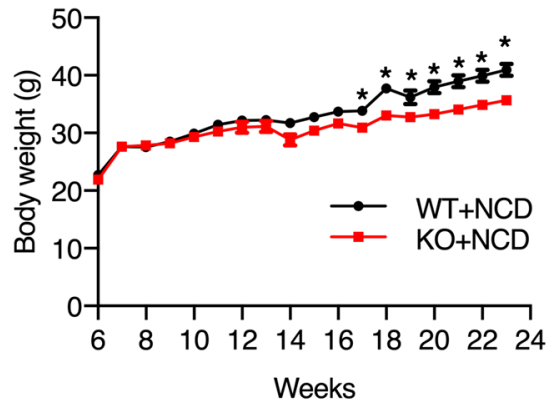

E

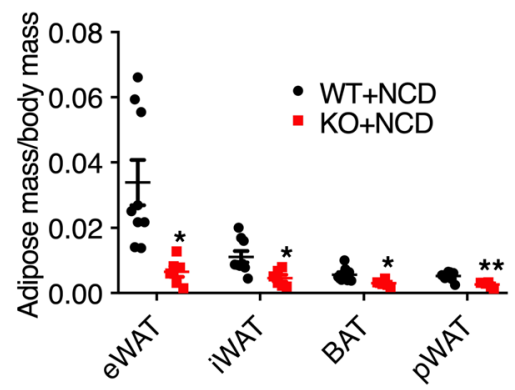

G

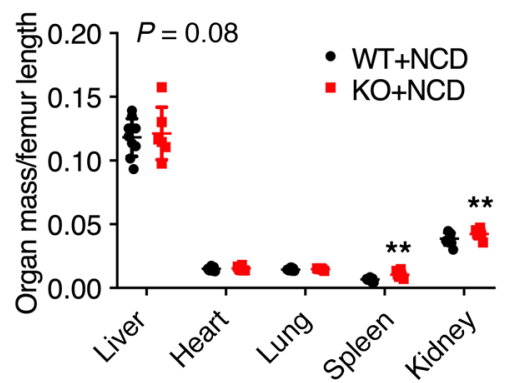

C
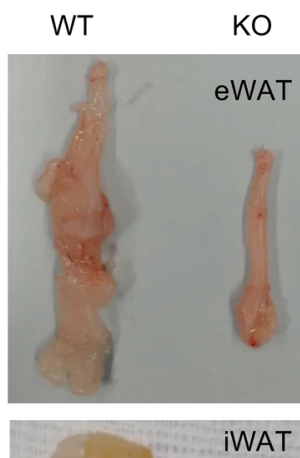

IWAT
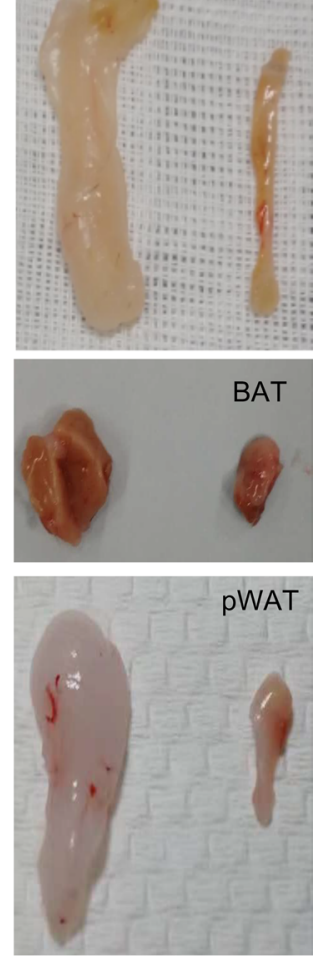

Figure 2. Deletion of kindlin-2 in adipocytes results in dramatically low adipose tissue mass in mice. (A) Representative image of male WT and KO mice. (B) The growth curve of WT and KO mice ( $N=8-10$ mice per group). (C) Representative images of BAT and WAT depots. (D and E) The weights of adipose and (F and $\mathbf{G}$ ) non-adipose tissues ( $N=6-10$ mice per group). eWAT, epididymal white adipose tissue; iWAT, inguinal white adipose tissue; BAT, brown adipose tissue; pWAT, perirenal white adipose tissue. ${ }^{*} P<0.05,{ }^{* *} P<0.01$ for KO vs. WT by Student's $t$ test.

WT mice (Supplemental Figure 2G). HFD feeding elevated the levels of circulating nonesterified fatty acids (NEFAs) and triglycerides (TGs) in KO mice compared with WT littermates (Figure 4, O and P, and Supplemental Figure 2, H and I). To determine the possible contributions of food intake to the changes in body weight and adiposity, food intake of the mice was also monitored. However, there was no marked difference in food intake between KO and WT groups (Figure 4Q and Supplemental Figure 2G).

Impaired lipid metabolism and massive fatty livers in HFD-fed KO mice. With the challenge of HFD, decreases in expression of adipogenesis genes, including those encoding Ppary, Cebpa, and Ap2, were observed in both WAT and BAT of KO mice compared with control littermates (Figure 5, A and B). Transcript levels of genes involved in lipogenesis, including those encoding Acc, Fas, and ATP citrate lyase (Acly), were also reduced in both WAT and BAT of HFD-fed KO mice compared with those in control littermates (Figure 5, A and B). Similarly, the expression levels of genes involved in lipolysis, including those encoding Atgl, Hsl, and adrenoceptor $\beta 3$ (Adrb3), were also reduced in both WAT and BAT in HFD-fed KO mice relative to those of HFD-fed WT mice (Figure 5, A and B).

Reduction of fatty acid oxidative transcript levels in WAT was also observed; the mRNA levels of Ppar $\alpha$, pyruvate dehydrogenase lipoamide kinase (Pdk4), and cell death activator (Cide $\alpha$ ) were decreased in 
A

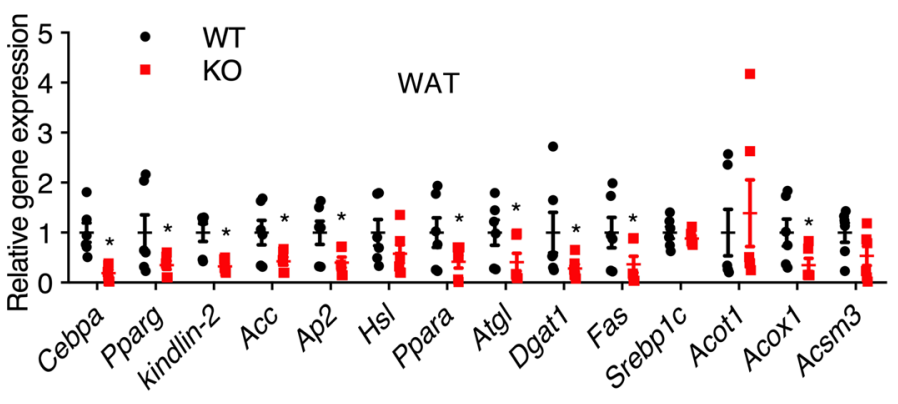

B

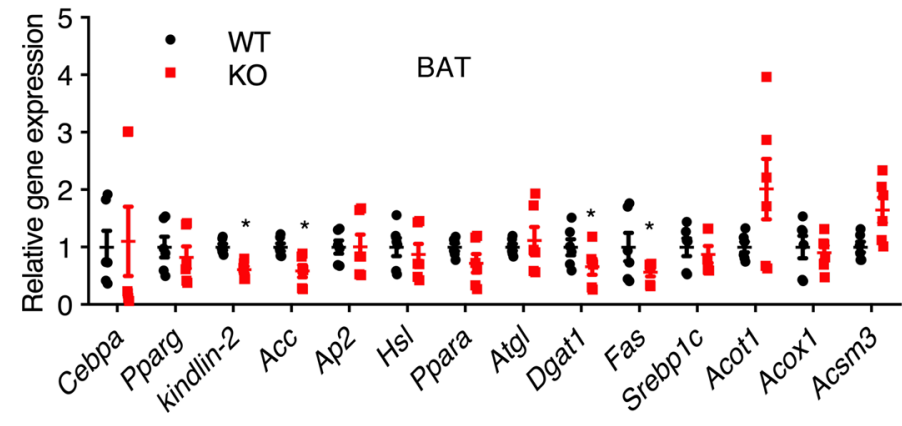

C
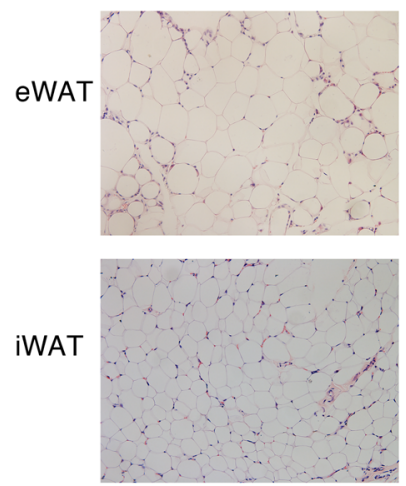

BAT

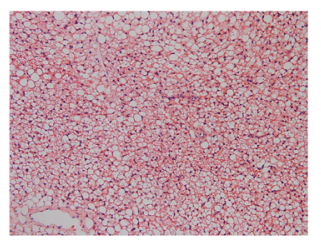

$\mathrm{KO}$
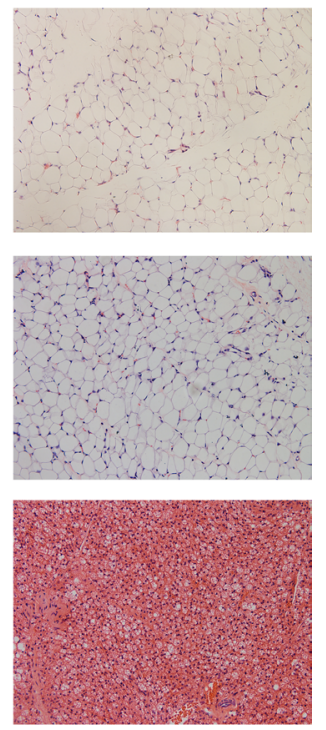

D

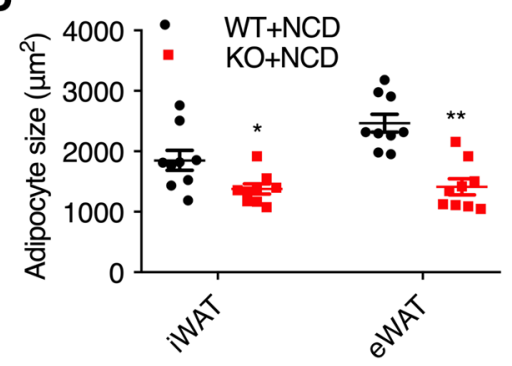

E

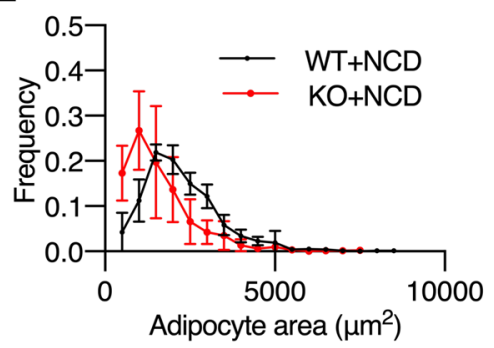

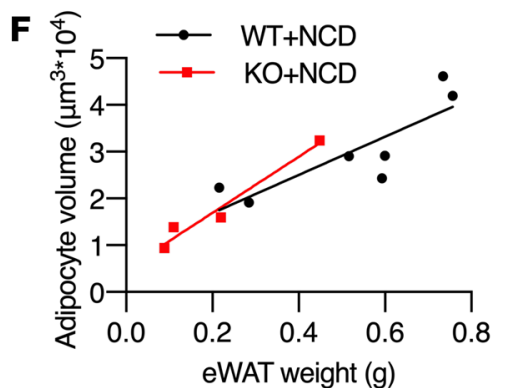

Figure 3. Kindlin-2 deficiency impairs adipocyte gene expression and differentiation. (A and $\mathbf{B}$ ) Real-time quantitative PCR analysis of adipogenic, thermogenic, and mitochondrial genes in (A) iWAT and (B) BAT from WT and KO mice ( $N=6$ mice per group). (C) Representative H\&E staining of eWAT, iWAT, and BAT of WT and KO mice (original magnification, $\times 200$ ). (D) Adipocyte size in adipose tissue. (E) Distribution of eWAT adipocyte size as determined by histomorphometric analyses ( $N=4-7$ mice per group). (F) Relationship between calculated mean adipocyte volume and estimated total adipocyte number in the eWAT based on H\&E staining in $\mathbf{C} .{ }^{*} P<0.05,{ }^{* *} P<0.01$ for KO vs. WT by Student's $t$ test.

WAT of HFD-fed KO mice compared with HFD-fed WT mice (Figure 5A). Alteration of adipocyte morphology was also obvious in KO adipose tissues. After HFD feeding, the size of KO adipocytes, including that of eWAT, iWAT, and BAT, was smaller than that of WT cells (Figure 5, C-E). The adipocyte number was reduced after HFD challenge (Figure 5F).

The findings described above suggest that decreases in transcript levels of adipogenesis-related genes and those involved in lipid oxidation and synthesis lead to elevated circulating blood lipid levels (Figure 4, O and $\mathrm{P})$. We next asked whether the higher levels of circulating lipids resulted in increased hepatic steatosis in KO mice. Histology of liver sections of KO mice revealed significant hepatic steatosis in KO mice (Supplemental Figure 3A), which was aggravated after HFD challenge (Figure 5G). Under the NCD condition, expression of Hsl, a lipolysis-related gene, was upregulated in KO liver (Supplemental Figure 3B); this might be caused by a negative feedback mechanism. With the HFD challenge, TG synthesis was increased, and lipolysis and fatty acid oxidation were reduced in KO liver (Supplemental Figure 4). Thus, KO mice fed with HFD have reduced expression of genes that regulate lipid metabolism and, consequently, increased circulating lipid levels, all of which might contribute to the hepatic steatosis and insulin resistance observed in the KO animals. In addition, increased macrophage infiltration was revealed by F4/80 staining in KO iWAT (Figure $5 \mathrm{H}$ and Supplemental Figure 5), which was consistent with the inflammatory effects of adipocyte death (14). 
A

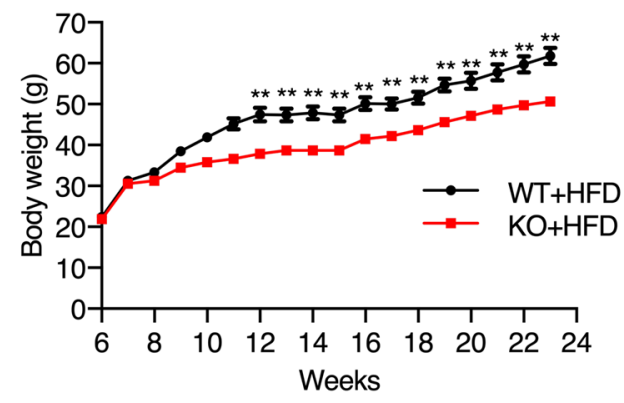

B
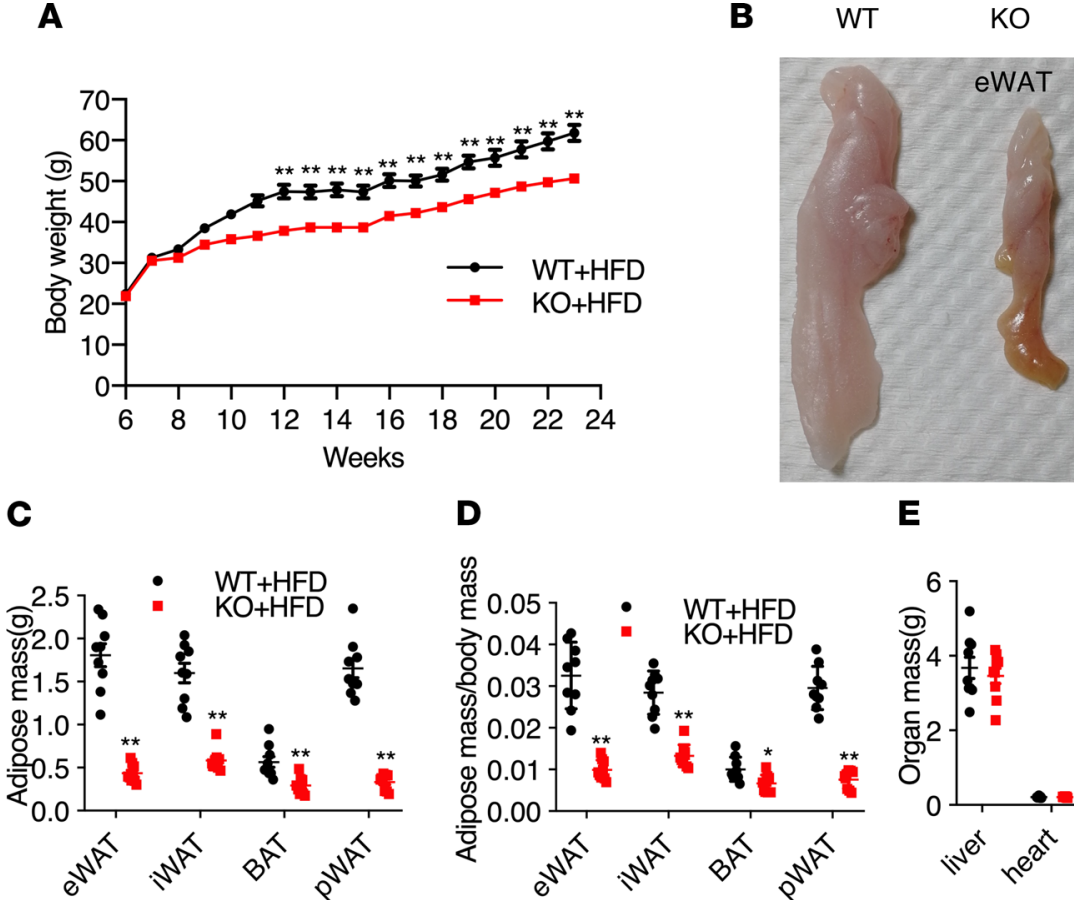

G

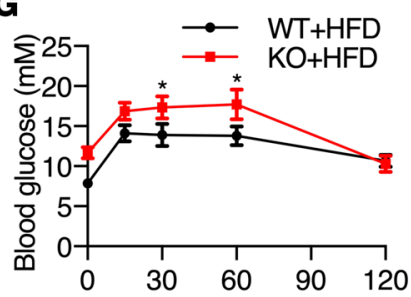

K

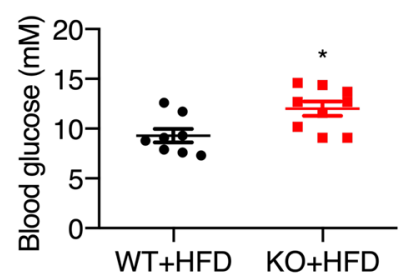

O

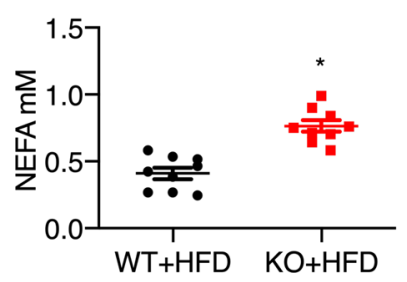

D

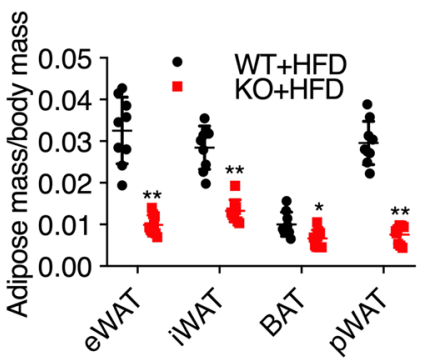

H

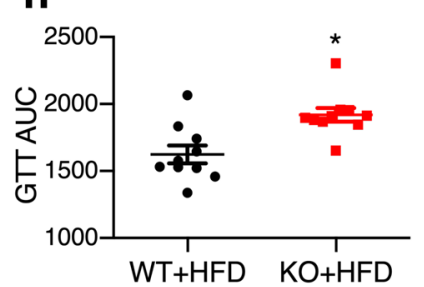

L

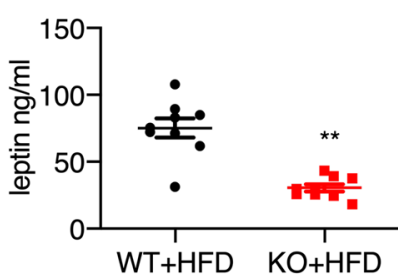

$\mathbf{P}$

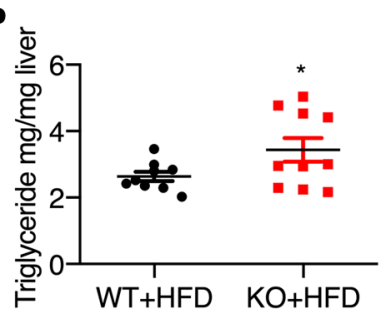

E
WT

KO

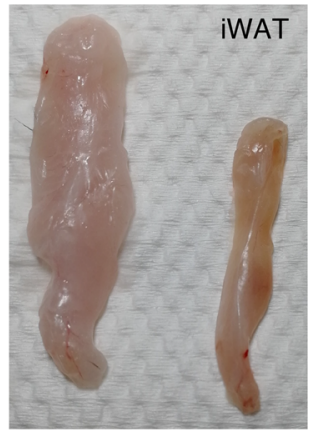

$\mathbf{F}$
WT KO
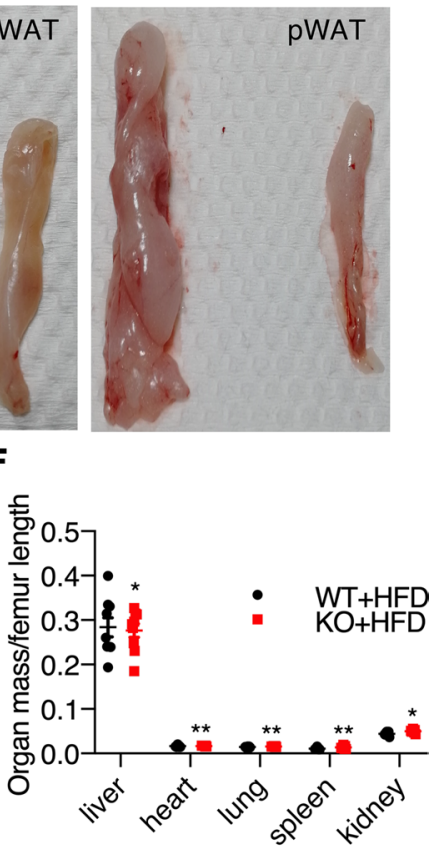

J

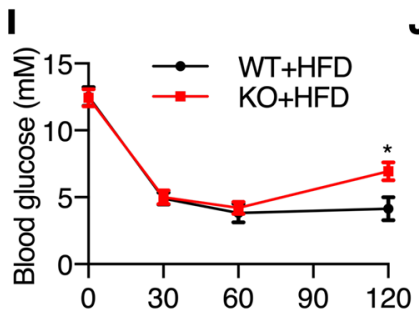

M

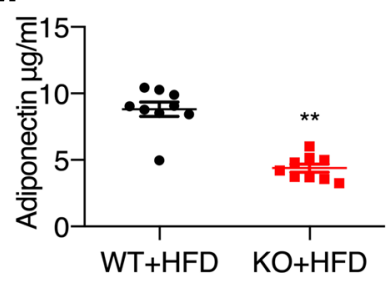

N

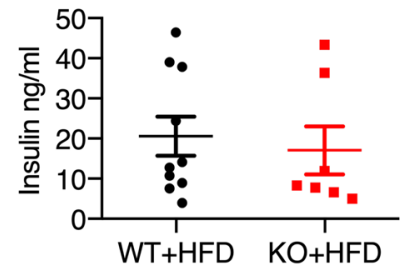

Figure 4. Elevated fasting blood glucose level, glucose intolerance, and peripheral insulin resistance in HFD-fed KO mice. (A) Body weight growth curve in WT and KO mice ( $N=10$ mice per group). (B) Representative images of adipose tissue depots. (C-F) The weights of adipose and non-adipose tissues ( $N=6-9$ mice per group). (G) Intraperitoneal glucose tolerance test (GTT) of WT and KO mice after 18 weeks of the HFD ( $N=9$ mice per group). (H) Area under the curve (AUC) for CTT. (I) Intraperitoneal insulin tolerance (ITT) test of WT and KO mice after 18 weeks on the HFD ( $N=9$ mice per group). (J) Area under the curve (AUC) for ITT. (K) Glucose, (L) leptin, (M) adiponectin, (N) insulin, (0) NEFA, and (P) TC levels in WT and KO mice after 18 weeks on the HFD ( $N=9$ mice per group). (Q) Food intake ( $N=9$ mice per group). ${ }^{*} P<0.05,{ }^{*} P<0.01$ for KO vs. WT by Student's $t$ test. 
A

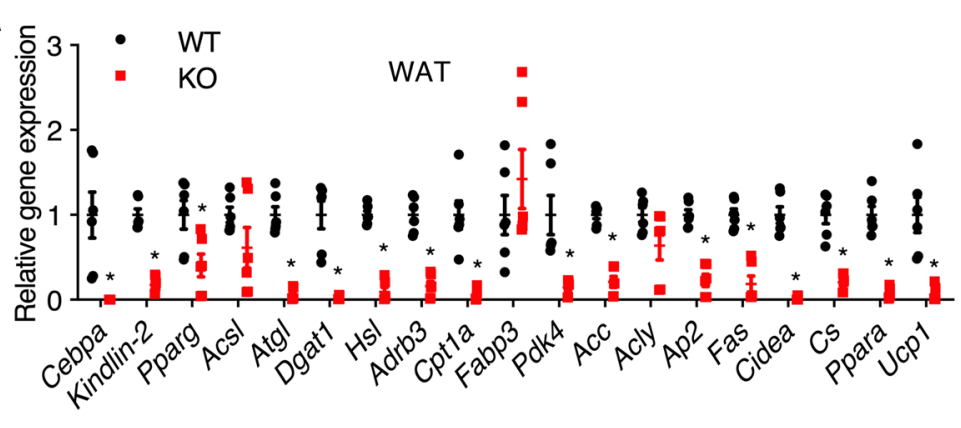

B

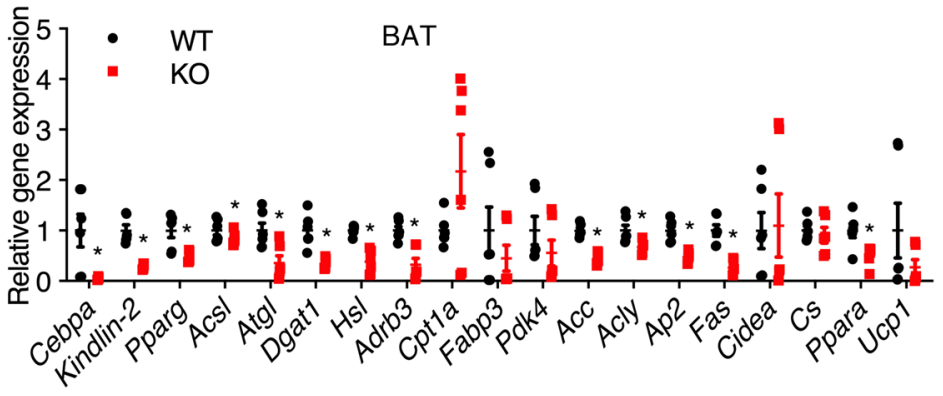

C

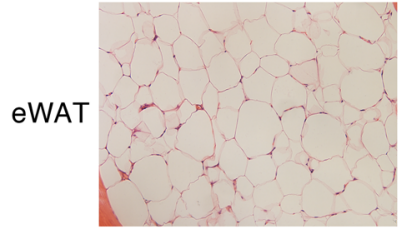

iWAT

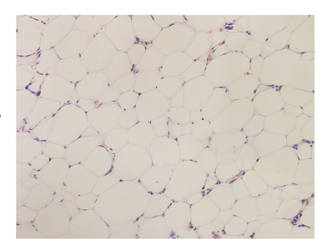

BAT

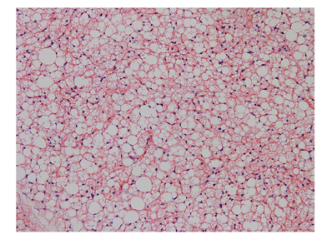

$\mathrm{KO}$
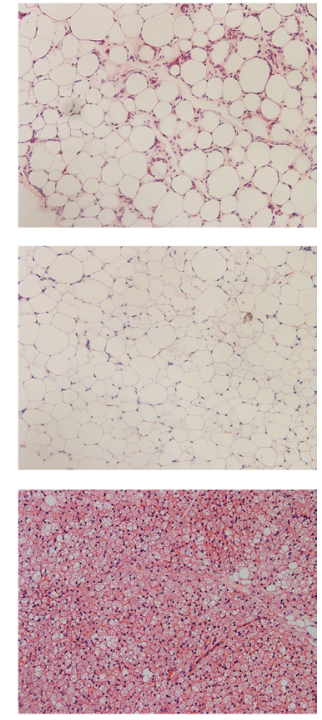

D

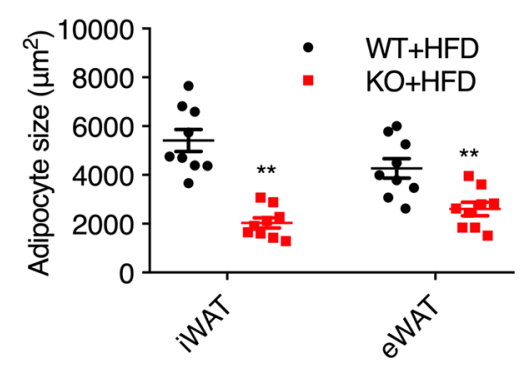

$\mathbf{G}$

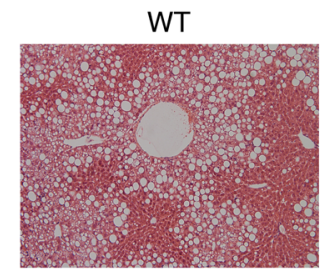

E
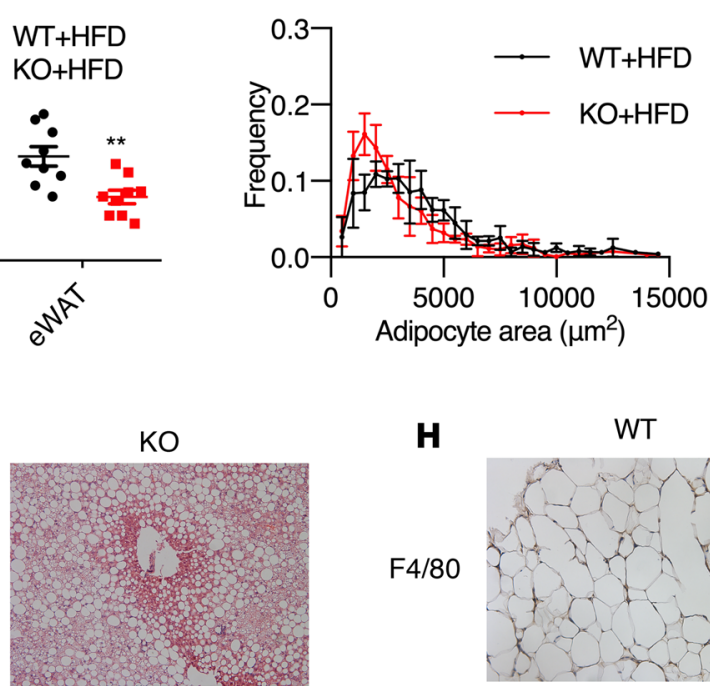

H

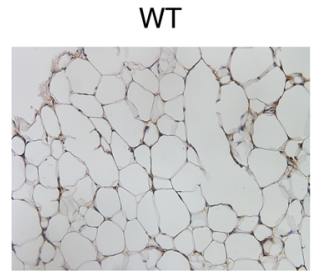

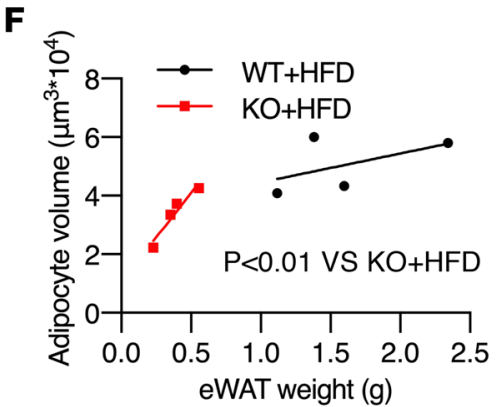

$\mathrm{KO}$

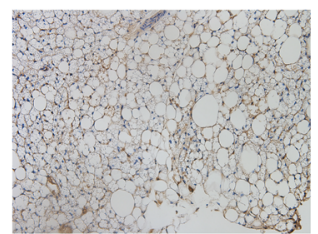

Figure 5. Deregulated lipid metabolism in HFD-fed KO mice. (A and B) Quantitative real-time reverse transcriptase PCR ( $q P C R$ ) analyses of gene expression in iWAT and BAT of HFD-fed WT and KO mice ( $N=6$ mice per group). (C) Representative H\&E staining of BAT, eWAT, iWAT, and BAT of WT and KO mice after 18 weeks of HFD feeding (original magnification, $\times 200$ ). (D) Adipocyte size in adipose tissue. (E) Distribution of eWAT adipocyte size as determined by histomorphometric analyses ( $N=4-7$ mice per group). (F) Relationship between calculated mean adipocyte volume and calculated total adipocyte number in the eWAT based on H\&E staining in C. (C) H\&E staining of livers from WT and KO mice after 18 weeks of HFD feeding (original magnification, $\times 200$ ). (H) Immunohistochemical staining of F4/80 in iWAT from WT and KO mice after 18 weeks of HFD feeding (original magnification, $\times 200$ ). ${ }^{*} P<0.05,{ }^{* *} P<0.01$ for KO vs. WT by Student's $t$ test.

Kindlin-2 loss impairs multiple key signaling pathways in adipocytes. Consistent with the reduced mRNA expression, the levels of adipogenesis-related proteins, including Cebp $\alpha$, Ppar $\gamma$, and Ap2, were all dramatically reduced in $\mathrm{KO}$ mice (Figure 6A). Previous studies have shown that deletion of AKT and mTOR resulted in lipoatrophy, suggesting that both factors are essential for development of the adipose tissue (15, 16). We next performed Western blotting and measured the protein levels of AKT and mTOR in adipose tissues of the both genotypes. The results showed that the levels of both total and phosphorylated AKT and mTOR proteins were all dramatically reduced in $\mathrm{KO}$ adipose tissues compared with those of WT adipose tissues (Figure 6A). Because $\beta$-catenin is critical for organ development (17), we next measured its protein 
A

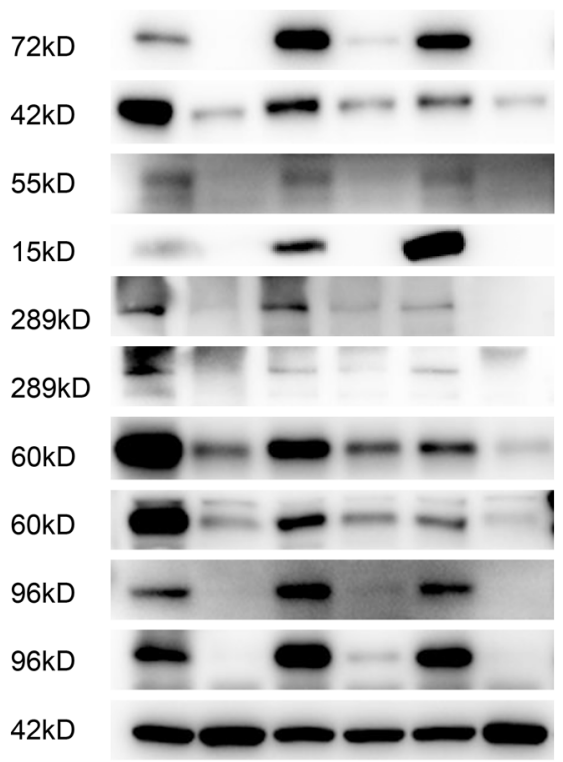

KINDLIN-2

CEBPa

PPARY

FABP4

p-mTOR

mTOR

p-AKT

AKT

Active- $\beta$-catenin

$\beta$-catenin

ACTIN
B
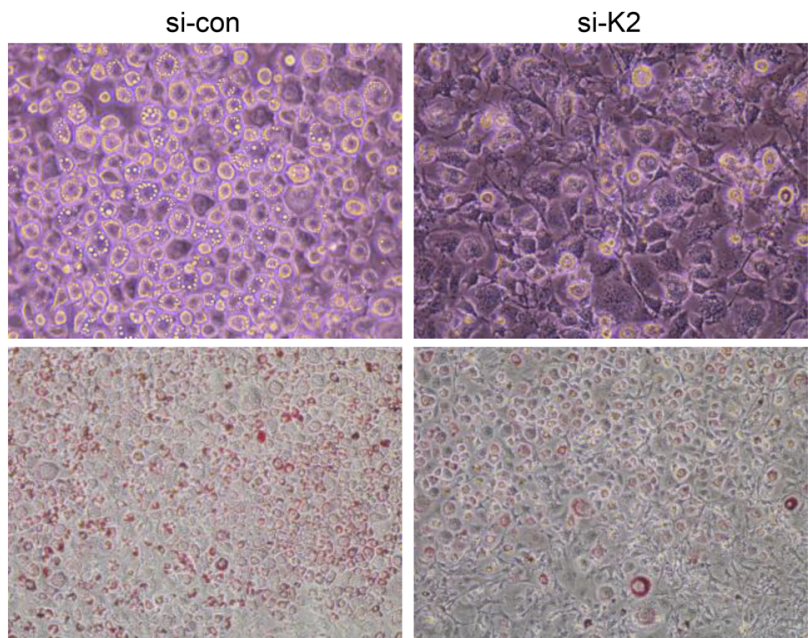

C

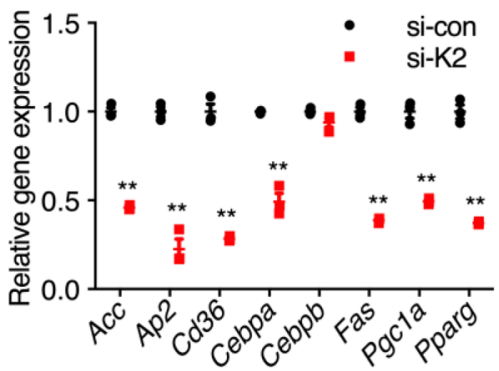

E
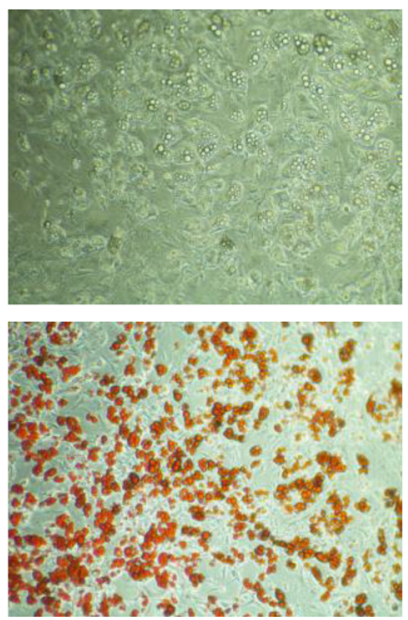

KO
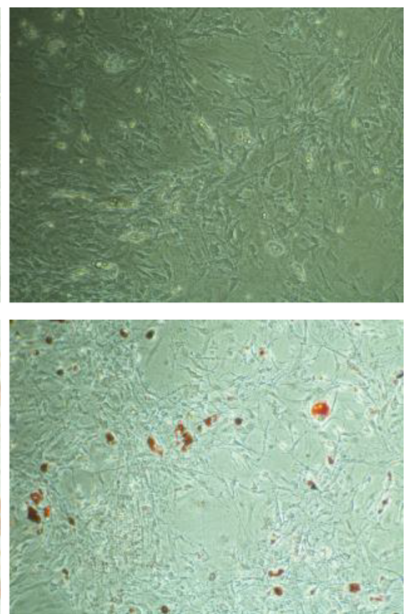

D

$15 \mathrm{kD}$

$42 \mathrm{kD}$

$55 \mathrm{kD}$

$72 \mathrm{kD}$

$42 \mathrm{kD}$

Figure 6. Deletion of kindlin-2 inhibits adipogenesis in vitro. (A) Western blot analysis of protein levels of adipogenesis genes in iWAT of WT and KO mice. (B) Oil Red O staining of 3T3-L1 cells treated with control siRNA or kindlin-2 siRNA (si-con and si-K2, respectively) after differentiation ( $N=3$ replicates). Original magnification, $\times 200$ (top) and $\times 100$ (bottom). (C) Quantitative real-time reverse transcriptase PCR (qPCR) analyses of mRNA levels of adipogenesis genes in si-con and si-K2 cells ( $N=3$ replicates). (D) Western blot analysis of adipogenesis protein expression in si-con and si-K2 cells ( $N=3$ replicates). (E) Oil Red $\mathrm{O}$ staining of WT and KO stromal vessel fraction (SVF) cells after differentiation. Original magnification, $\times 200 .{ }^{*} P<0.05,{ }^{*} P<$ 0.01 for KO vs. WT by Student's $t$ test.

expression level in adipose tissue and found dramatic reductions in both total and phosphorylated $\beta$-catenin in $\mathrm{KO}$ adipose tissue compared with that of WT adipose tissue (Figure 6A). To verify whether deletion of kindlin-2 affects preadipocyte differentiation in vitro, we cultured 3T3-L1 preadipocytes and differentiated them into mature adipocytes. During in vitro adipocyte differentiation, kindlin-2 expression was markedly upregulated on day 4; this upregulation was then decreased on day 6 (Supplemental Figure 6). As indicated by Oil Red O staining, knockdown of kindlin-2 expression inhibited adipogenesis of 3T3-L1 cells (Figure $6 \mathrm{~B})$. In line with our in vivo results, knockdown of kindlin-2 expression significantly reduced the expression levels of all adipogenesis-related genes in differentiated 3T3-L1 cell cultures (Figure 6, C and D). The stromal-vascular fraction (SVF) of inguinal adipose tissue was isolated from WT and KO mice and treated 
A

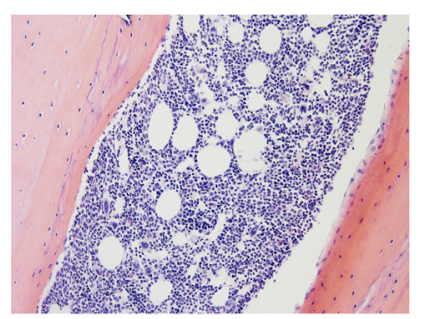

KO

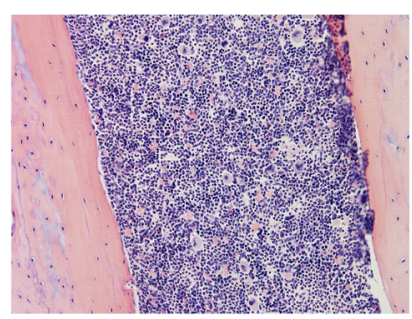

B
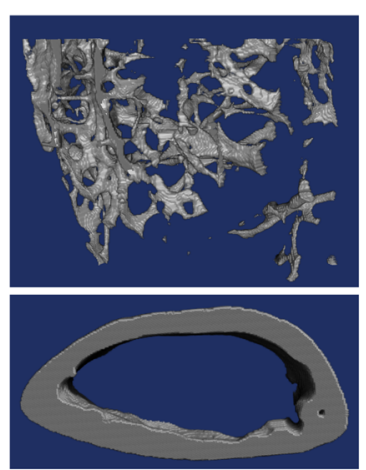

$\mathrm{KO}$
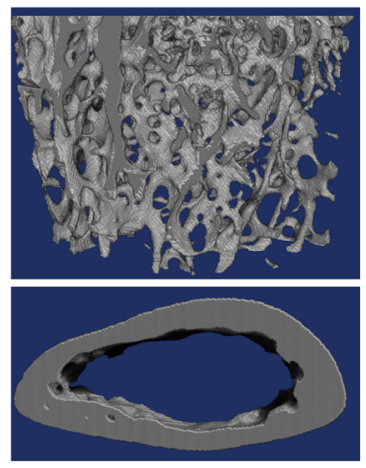

C

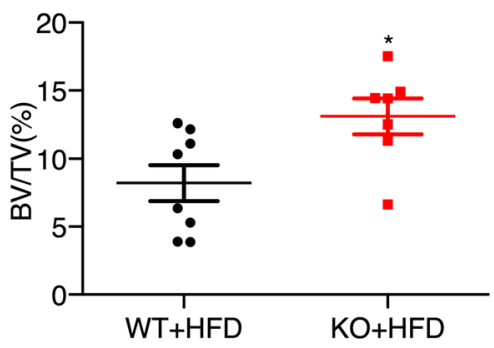

F

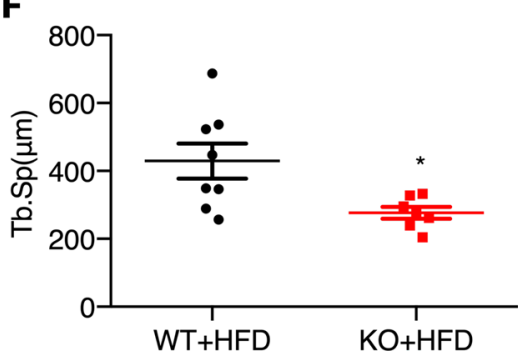

D

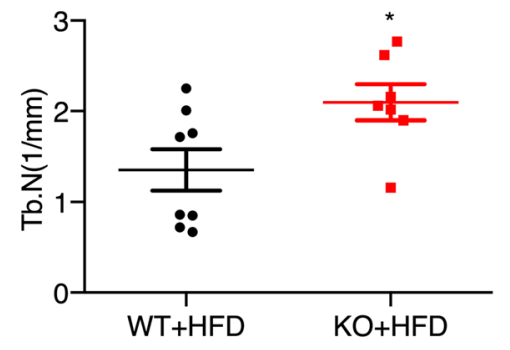

G

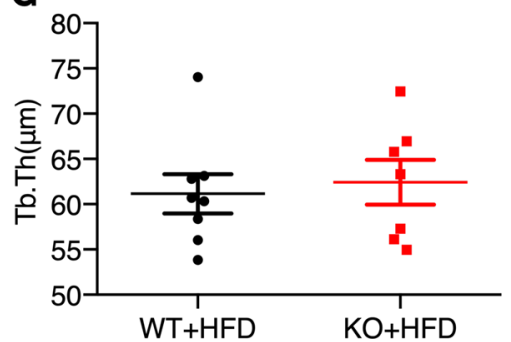

E

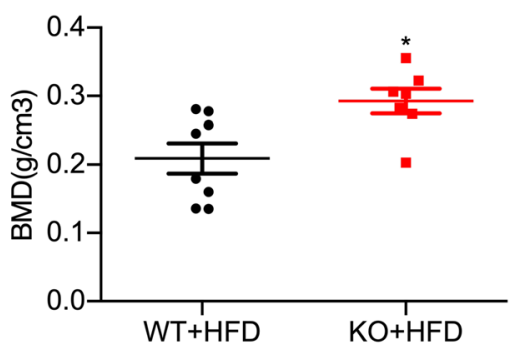

H

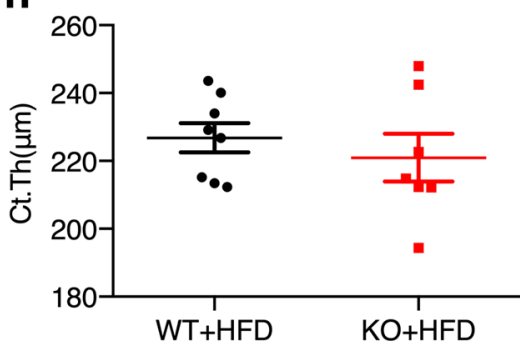

Figure 7. Deleting kindlin-2 in adipocytes causes high bone mass in KO mice. (A) H\&E staining of sections of tibia from WT and KO mice after 18 weeks HFD feeding ( $N=4$ mice per group). Original magnification, $\times 200$. (B) Three-dimensional (3D) reconstruction from micro-computerized tomography ( $\mu C T$ ) scans of femurs from WT and KO mice after 18 weeks HFD feeding. (C-H) Bone histomorphometric analyses ( $N=7-8$ mice per group). (C) Bone volume fraction (BV/TV), (D) trabecular number (Tb.N), (E) bone mineral density (BMD), (F) trabecular separation (Tb.Sp), (G) trabecular thickness (Tb.Th), and (H) cortical bone thickness (Cort.Th). ${ }^{*} P<0.05$ for KO vs. WT by Student's $t$ test.

to induce adipogenic differentiation in vitro. Results from Oil Red O staining revealed that kindlin-2 loss inhibited SVF differentiation toward adipocytes (Figure 6E). Collectively, these results demonstrate that kindlin-2 is essential for adipocyte differentiation in vitro and in vivo.

HFD-fed Kindlin-2-KO mice display a high bone mass phenotype. Osteoblasts and adipocytes are both derived from mesenchymal stem cells and interactions between fat and bone are vital for normal bone integrity (18). $\mathrm{H} \& \mathrm{E}$ staining of femur sections revealed that bone marrow adiposity was markedly reduced in KO relative to WT mice (Figure 7A). To investigate whether kindlin-2 loss in adipocytes impacts bone mass, we performed micro-computerized tomography $(\mu \mathrm{CT})$ analysis of the femurs and found increased bone mass in $\mathrm{KO}$ mice compared with control littermate (Figure 7B). Quantitative analysis confirmed that KO mice displayed dramatic increases in bone volume fraction (BV/TV; Figure 7C), trabecular number (Tb.N; Figure 7D), and bone mineral density (BMD; Figure 7E) and decrease in trabecular separation (Tb.Sp; Figure 7F). KO mice had a noticeable decrease in Tb.Sp, without a significant difference in the values of trabecular thickness (Tb.Th; Figure 7G) and cortical bone thickness (Cort.Th; Figure 7H). However, there were no major differences between WT and KO mice under the NCD condition (Supplemental Figure 7). The BMD and Ct.Th were slightly, but significantly, reduced in NCD-treated KO mice compared with those in NCD-treated WT mice (Supplemental Figure 7). Taken together, these results suggest that adipocyte kindlin-2 signaling is critical for bone homeostasis. 


\section{Discussion}

In the present study, we demonstrate, for the first time to our knowledge, a critical role of kindlin- 2 in regulation of adipocyte formation and differentiation and lipids and glucose metabolisms. Thus, adipocyte-specific kindlin-2 ablation leads to severe lipoatrophy, hyperlipidemia, massive fatty liver, glucose intolerance, and insulin resistance in mice. Furthermore, adipocyte kindlin-2 indirectly regulates bone mass.

The lipodystrophic syndrome observed in the KO mice is similar to human generalized lipodystrophy in several ways. For example, both are characterized by low leptin levels, marked insulin resistance, hyperlipidemia, and fatty liver disease. Lipoatrophy reduces the capacity of adipose tissue to take up and store additional TGs, which will cause lipid redistribution that is associated with systemic insulin resistance and hepatic steatosis (19). Adipose-specific deletion of several other factors induced similar lipoatrophy and hepatic steatosis $(15,16,20-24)$. For example, mice with adipose-specific deletion of the insulin receptor $(21,22)$, Raptor/mTORC1 (16), AKT (23), or LMNA (24) developed significant lipodystrophy and hepatic steatosis. Interestingly, LMNA mutation caused significant BAT "whitening" (24); this is not observed in Kindlin-2-KO mice. Congenital and acquired lipodystrophies could cause insulin resistance and hepatic steatosis $(25,26)$. Importantly, results of the present study provide the first demonstration to our knowledge that adipocyte kindlin- 2 signaling plays a pivotal role in control of adipose tissue mass.

We demonstrated that the KO mice fed on NCD did not display any glucose intolerance, even though they had dramatically reduced expression of genes involved in adipogenesis and thermogenic regulation. Interestingly, when fed on HFD, these animals exhibited marked glucose intolerance and insulin resistance. This observed deregulation of glucose homeostasis in HFD-fed mutant mice was evident with decreases in body weight and adiposity and with increases in circulating lipids. The potential explanation for the increased blood lipid level observed in the mutant mice is related to reduced expression of genes involved in lipid breakdown, lipid uptake, and fatty acid oxidation, which all reduce clearance of circulation lipids by adipose tissue. Evidence has shown that the mobilization of BAT increases the clearance of lipids in both humans and mice $(27,28)$. In addition, dysregulated expression of lipogenesis genes leads to disturbed lipid storage and causes insulin resistance (29). Notably, our ITT results showed nearly identical values for the WT and KO mice at the early time points (30,60, and 90 minutes) and only a significant difference at the 120 -minute end time point. These findings support the notion that insulin sensitivity is similar in $\mathrm{KO}$ and WT animals, but that the counter-regulatory mechanisms (epinephrine, norepinephrine, glucocorticoids) to increase circulating glucose might be impaired in $\mathrm{KO}$ mice. Future study will explore this possibility.

In the present study we observed an increased inflammation of adipose tissue in the mutant mice. The inflammation and macrophage infiltration in adipose tissue contribute to the enhanced lipolysis and elevated circulating lipids, which causes insulin resistance, as suggested by Wu and coworkers $(19,22)$.

The increased blood lipid levels contribute to, at least in part, the insulin resistance and fatty liver phenotype observed in the mutant mice. Hyperlipidemia is closely associated with insulin resistance and hepatic steatosis (30). Kindlin-2 deficiency-induced reduction in adipokine production also contributes to the dysregulation of glucose homeostasis observed in the mutant mice. This notion is supported by results from a previous study demonstrating that decreased adiponectin expression is correlated with insulin resistance. In obese mice, adiponectin decreases insulin resistance and improves insulin sensitivity by decreasing TG content in liver and muscle (31).

Reduced expression of both mTOR and AKT induced by kindlin-2 deficiency could contribute to the low adipose tissue mass phenotype of the mutant mice because both pathways are known to be critical for adipose tissue development $(15,23)$. Results from in vitro studies showed that AKT signaling is necessary for adipogenesis (32). AKT1 is required for adipogenesis in 3T3L1 preadipocytes and mouse embryonic fibroblasts, while AKT2 is dispensable (33-35). Mechanistically, AKT functions in adipocyte precursor cells to suppress FoxO1 activity (36). The role of mTOR in adipose tissues has been well studied by genetic perturbation of key components of this pathway $(15,37,38)$. Deletion of mTOR decreased lipid accumulation and inhibited adipocyte differentiation by the Ppary signaling pathway (15). Molecular mechanism(s) underlying the downregulation of AKT and mTOR in adipocytes mediated by kindlin-2 loss remain to be determined. Numerous studies have shown that Wnt/ $\beta$-catenin signaling is important for multiple organs' development $(16,39)$. While several in vitro studies showed that pharmacological manipulation of adipogenesis altered expression of $\beta$-catenin $(40,41)$, there is no direct in vitro and in vivo evidence to our knowledge that $\beta$-catenin regulates adipogenesis. Yu et al. reported that kindlin- 2 formed a transcriptional complex with $\beta$-catenin to regulate Wnt signaling (42). Results of the present study revealed that kindlin-2 
loss drastically reduced the levels of both active and total $\beta$-catenin protein levels. Thus, it is possible that kindlin- 2 regulates adipogenesis by modulating, in part, the Wnt/ $\beta$-catenin signaling pathway. Future study will explore this possibility.

The dramatic increases in bone mass and bone mineral density observed in the mutant mice suggest an intrinsic role of adipocyte kindlin-2 signaling in control of bone mass. Osteoblasts and adipocytes are both derived from mesenchymal precursors $(18,43)$. Our previous study showed that deletion of kindlin-2 in mesenchymal lineage cells inhibited chondrogenesis and impaired skeletal development (10). Dramatically reduced production of leptin, a potent inhibitor of bone formation $(44,45)$, contributes, at least in part, to the high bone mass phenotype of the mutant mice. Adiponectin, an adipocyte-derived cytokine, exerts an inhibitory effect on bone metabolism $(46,47)$. Because kindlin-2 loss dramatically reduced the blood levels of adiponectin (Figure $4 \mathrm{M}$ ), it is possible that the reduction in adiponectin also contributes to the high bone mass phenotype of the mutant mice. It is known that TNF- $\alpha$ reduces bone mass (48). It will be interesting to determine whether kindlin-2 loss decreases TNF- $\alpha$ production in the mutant mice.

In summary, the striking lipodystrophic phenotype of the kindlin-2-mutant mice demonstrates an essential requirement for kindlin-2 expression in adipogenesis. Results of the present study also demonstrate the importance of maintaining a proper adipose tissue mass, given the fact that the lipodystrophic phenotype caused by kindlin-2 deficiency results in severe hyperlipidemia, massive fatty liver, type II diabetes-like phenotypes, and high bone mass.

\section{Methods}

Animal studies. Generation of Kindlin-2 $2^{f / f l}$ mice, in which loxP sites were inserted from exons 5 and 6 at the Kindlin-2 locus through homologous recombination, was described in our previous study (10). To delete kindlin-2 expression in adipocytes, we first bred the Kindlin-2 $2^{f / \AA}$ mice with the Adipoq-Cre-transgenic mice harboring the Adipoq-Cre BAC transgene expressing Cre recombinase under control of the mouse adiponectin (Adipoq) promoter/enhancer regions within the BAC transgene (purchased from the Jackson Laboratory; ref. 13) and obtained the Kindlin-2 $2^{f /+}$ Adipoq-Cre mice. Further breeding of the Kindlin-2 $2^{f /+}$ Adipoq-Cre mice with Kindlin-2 $2^{f / f l}$ mice resulted in the adipocyte conditional knockout mice, i.e., the Kindlin-2 $2^{f / f l}$ Adipoq-Cre mice, i.e., the adipocyte-selective Kindlin-2-KO mice. The Cre-negative floxed Kindlin-2 mice (i.e., Kindlin$\left.2^{f / f f}\right)$ and Cre-transgenic mice (i.e., Adipoq-Cre) were used as controls in this study. All mice used in this study have been crossed with normal C57BL/ 6 mice for at least 10 generations. For consistency and to minimize use of animals, only male mice were used for all experiments in this study. Mice were group-housed at $20^{\circ} \mathrm{C}$ to $24^{\circ} \mathrm{C}$ and exposed to a 12-hour light/12-hour dark cycle. For induction of obesity and insulin resistance, 6-week-old mice were fed with an HFD (60\% fat diet, measured in kcal, catalog D12492; Research Diets) for 18 weeks. Mice fasted for 16 hours were injected with glucose $(1 \mathrm{~g} / \mathrm{kg}$ body weight, i.p.) for the GTT and mice fasted for 4 hours were injected with insulin ( 0.5 units of insulin $/ \mathrm{kg}$ body weight, i.p.) for the ITT. Blood glucose and insulin levels were measured from tail vein blood collected at the indicated times.

Real-time quantitative RT-PCR analysis. Total RNA was extracted from tissues and cells using TRIzol reagent (Invitrogen, 15596018) according to the manufacturer's instructions (49). Total RNA ( $2 \mu \mathrm{g})$ from each sample was reverse transcribed using the cDNA Reverse Transcription Kit (Applied Biosystems, 4374966) according to the manufacturer's instructions (50). Samples were normalized to Gapdh mRNA expression. The DNA sequences of primers used for qPCR analyses are summarized in Supplemental Table 1.

Western blot analysis. Western blot analyses on $30 \mu \mathrm{g}$ of protein extracts from cells and tissues were performed as previously described (10). Briefly, frozen tissues were homogenized in RIPA lysis buffer containing protease inhibitor cocktail (Thermo Fisher Scientific, 78437). Protein concentrations were determined by BCA assay (Bio-Rad, 2634). SDS-PAGE was performed using approximately $30 \mu \mathrm{g}$ of protein which was transferred to a PVDF membrane. The membrane was blocked in 5\% nonfat milk in Tris-buffered saline/Tween 20 buffer, probed with primary antibodies, followed by incubation with secondary antibodies conjugated with horseradish peroxidase, and visualized using a Western Blotting Detection Kit (Bio-Rad, 1705062). Western blots were probed with the primary antibodies at a dilution of 1:1,000 except for the following antibodies: anti- $\beta$-actin (Zhongshan Jinqiao Biotechnology, TA-09, 1:5,000), anti-Fabp4 (Abcam, ab92501, 1:2000) and anti-kindlin-2 (Millipore, MAB2617, 1:2000). Anti-Cebpa (catalog 2295), anti- $\beta$-catenin (catalog, 9562), anti-Ppary (catalog 2443), anti-active- $\beta$-catenin (catalog 8814), anti-Hsl (catalog 4107), anti-mTOR (catalog 2983), anti-p-mTOR (catalog 2971), anti-AKT (catalog 4691), and anti-p-AKT (catalog 4060) antibodies were purchased from Cell Signaling Technology. 
Histological analysis. The adipose pads, including eWAT, iWAT, BAT, and pWAT, were isolated from mice and stained with $H \& E$ for morphological analysis $(10,50)$. Adipose tissues from mice were fixed for 48 hours with $10 \%$ formalin, dehydrated, and embedded in paraffin to measure the macrophage infiltration. After blocking with PBS containing 1\% BSA, sections were subjected to immunohistochemical staining overnight at $4^{\circ} \mathrm{C}$ with a 1:150 dilution of a rat monoclonal antibody against F4/80 (Cell Signaling Technology, catalog 70076). A biotin-conjugated rabbit polyclonal against rat IgG (Cell Signaling Technology, catalog 3900) was used at 1:100 dilution as the secondary antibody. Slides were observed and imaged with a light microscope equipped with a digital camera. Using ImageJ software (NIH), adipocyte volume was calculated from H\&E-stained paraffin sections as described previously (51). Adipocyte number was calculated by dividing the epididymal adipose tissue volume by adipocyte volume.

Isolation of SVF from WAT and in vitro differentiation. SVF cells were isolated from WAT as previously reported (52). Briefly, WAT depots were minced and digested with collagenase D (Roche, 1108874103). Digestion was terminated by adding ice-cold DMEM plus $10 \%$ FBS, followed by centrifugation and filtration through $70-\mu \mathrm{m}$ cell strainers. SVF cells were plated at $1 \times 10^{6}$ cells per well in 6-well plates and grown in DMEM supplemented with 10\% FBS. After 2 days, the cells were cultured in differentiation medium as previously described (52). 3T3-L1 cells (purchased from ATCC) were cultured in DMEM with $10 \%$ FBS. For adipogenic differentiation, 3T3-L1 cells were first cultured in differentiation media (DMEM, 10\% FBS, $5 \mu \mathrm{g} / \mathrm{mL}$ insulin, $2 \mu \mathrm{g} / \mathrm{mL}$ dexamethasone, and $0.5 \mathrm{mM} 3$-isobutyl-methylxanthine [IBMX]) for 2 days. Cells were then switched to media (DMEM, 10\% FBS and $5 \mu \mathrm{g} / \mathrm{mL}$ insulin) for 6 days. Media were changed every 2 days.

Oil Red O staining. In brief, following fixation of the mature adipocytes in $10 \%$ (v/v) formaldehyde solution for 30 minutes, the cells were stained with $0.5 \%$ Oil Red $\mathrm{O}$ for 30 minutes at room temperature, rinsed in PBS, as previously described (49).

Serum analysis. Blood samples were collected from 18 -week-old mice and kept at room temperature for 30 minutes. Samples were then centrifuged at 12,000 $\mathrm{g}$ for 10 minutes and the separated sera were collected. Insulin (Millipore, EARMI-13BK), adiponectin (R\&D Systems, MRP300), and leptin (R\&D Systems, MOB00) analyses were performed using commercially available ELISA kits according to the manufacturers' instructions. Plasma and liver TG (Nanjing Jiancheng, A010017) levels and plasma NEFAs (Nanjing Jiancheng, A042-2) were measured by colorimetric assay kits according to the manufacturer's instructions.

Liver TG assay. Frozen liver tissue samples were weighed and then homogenized in $1 \mathrm{~mL}$ of PBS. Samples were kept on ice the entire time. Liver lysates were centrifuged at 12,000 $g$ for 10 minutes at $4^{\circ} \mathrm{C}$. The supernatant was collected, and TG levels were determined using the Triglyceride Assay (Thermo Fisher Scientific, TR22421) according to the manufacturer's instructions. TG levels were normalized to individual tissue weights.

Body weight and food intake. Weekly body weight was measured on a NCD or HFD over a period of 18 weeks until the end of the experimental protocol. Mice were individually housed for 1 week before food intake measurement. The food intake data were collected for a 2-week period.

$\mu C T$ analysis. Fixed, nondemineralized femurs were used for $\mu \mathrm{CT}$ analysis in the Department of Biology of Southern University of Science and Technology using a Bruker $\mu$ CT (SkyScan 1172 Micro-CT) following the standards of techniques and terminology recommended by the American Society for Bone and Mineral Research (ASBMR; ref. 53).

Statistics. The sample size for each experiment was determined based on our previous experience. No randomization or blinding was applied for all our studies. Results are expressed as mean \pm SEM. All data were analyzed by an unpaired 2-tailed Student's $t$ test. Differences with $P<0.05$ were considered statistically significant.

Study approval. All animal studies were approved by the Institutional Animal Care and Use Committee (IACUC) of Southern University of Science and Technology.

\section{Author contributions}

HG, HC, and GX designed the study. HG, YG, QY, WY, RL, HC, CL, XB, DC, and GX conducted the study and collected data, and were responsible for data analysis and interpretation. HG and GX drafted the manuscript. GX, HC, and HG take the responsibility for the integrity of the data analysis. 


\section{Acknowledgments}

This work was supported, in part, by the National Natural Science Foundation of China grants (8163066 and 81870532), the Guangdong Provincial Science and Technology Innovation Council grant (2017B030301018), and Science and Technology Innovation Commission of Shenzhen Municipal Government grants (JCYJ20180302174246105, JCYJ20180302174117738, JSGG20180503182321166, and KQJSCX20180319114434843).

Address correspondence to: Guozhi Xiao, Rm 341, Faculty Research Building 1, Southern University of Science and Technology, 1088 Xueyuan Rd, Shenzhen 518055, China. Phone: 0755.88018488; Email: xiaogz@sustc.edu.cn. Or to: Huiling Cao, Rm 312, Faculty Research Building 1, Southern University of Science and Technology, 1088 Xueyuan Rd, Shenzhen 518055, China. Phone: 0755.88018408; Email: caohl@sustc.edu.cn.

1. Cannon B, Nedergaard J. Brown adipose tissue: function and physiological significance. Physiol Rev. 2004;84(1):277-359.

2. Rosen ED, Spiegelman BM. Adipocytes as regulators of energy balance and glucose homeostasis. Nature. 2006;444(7121):847-853

3. Ouchi N, Parker JL, Lugus JJ, Walsh K. Adipokines in inflammation and metabolic disease. Nat Rev Immunol. 2011;11(2):85-97.

4. Guilherme A, Virbasius JV, Puri V, Czech MP. Adipocyte dysfunctions linking obesity to insulin resistance and type 2 diabetes. Nat Rev Mol Cell Biol. 2008;9(5):367-377.

5. Mathieu P, Lemieux I, Després JP. Obesity, inflammation, and cardiovascular risk. Clin Pharmacol Ther. 2010;87(4):407-416.

6. Qatanani M, Lazar MA. Mechanisms of obesity-associated insulin resistance: many choices on the menu. Genes Dev. 2007;21(12):1443-1455.

7. Garg A. Lipodystrophies: genetic and acquired body fat disorders. J Clin Endocrinol Metab. 2011;96(11):3313-3325.

8. Huang-Doran I, Sleigh A, Rochford JJ, O'Rahilly S, Savage DB. Lipodystrophy: metabolic insights from a rare disorder. J Endocrinol. 2010;207(3):245-255.

9. Ussar S, Wang HV, Linder S, Fässler R, Moser M. The Kindlins: subcellular localization and expression during murine development. Exp Cell Res. 2006;312(16):3142-3151.

10. Wu C, et al. Kindlin-2 controls TGF- $\beta$ signalling and Sox9 expression to regulate chondrogenesis. Nat Commun. 2015;6:7531.

11. Sun Y, et al. Kindlin-2 association with Rho GDP-dissociation inhibitor . J Am Soc Nephrol. 2017;28(12):3545-3562.

12. Guo L, et al. Kindlin-2 regulates mesenchymal stem cell differentiation through control of YAP1/TAZ. J Cell Biol. 2018;217(4):1431-1451.

13. Lee KY, et al. Lessons on conditional gene targeting in mouse adipose tissue. Diabetes. 2013;62(3):864-874.

14. Pajvani UB, et al. Fat apoptosis through targeted activation of caspase 8: a new mouse model of inducible and reversible lipoatrophy. Nat Med. 2005;11(7):797-803.

15. Shan T, Zhang P, Jiang Q, Xiong Y, Wang Y, Kuang S. Adipocyte-specific deletion of mTOR inhibits adipose tissue development and causes insulin resistance in mice. Diabetologia. 2016;59(9):1995-2004.

16. Lee PL, Tang Y, Li H, Guertin DA. Raptor/mTORC1 loss in adipocytes causes progressive lipodystrophy and fatty liver disease. Mol Metab. 2016;5(6):422-432.

17. Tan X, Behari J, Cieply B, Michalopoulos GK, Monga SP. Conditional deletion of beta-catenin reveals its role in liver growth and regeneration. Gastroenterology. 2006;131(5):1561-1572.

18. Kawai M, Devlin MJ, Rosen CJ. Fat targets for skeletal health. Nat Rev Rheumatol. 2009;5(7):365-372.

19. Xia B, Cai GH, Yang H, Wang SP, Mitchell GA, Wu JW. Adipose tissue deficiency of hormone-sensitive lipase causes fatty liver in mice. PLoS Genet. 2017;13(12):e1007110.

20. Wang F, Mullican SE, DiSpirito JR, Peed LC, Lazar MA. Lipoatrophy and severe metabolic disturbance in mice with fat-specific deletion of PPAR $\gamma$. Proc Natl Acad Sci USA. 2013;110(46):18656-18661.

21. Qiang G, et al. Lipodystrophy and severe metabolic dysfunction in mice with adipose tissue-specific insulin receptor ablation. Mol Metab. 2016;5(7):480-490.

22. Softic S, et al. Lipodystrophy due to adipose tissue-specific insulin receptor knockout results in progressive NAFLD. Diabetes. 2016;65(8):2187-2200.

23. Shearin AL, Monks BR, Seale P, Birnbaum MJ. Lack of AKT in adipocytes causes severe lipodystrophy. Mol Metab. 2016;5(7):472-479.

24. Wojtanik KM, et al. The role of LMNA in adipose: a novel mouse model of lipodystrophy based on the Dunnigan-type familial partial lipodystrophy mutation. J Lipid Res. 2009;50(6):1068-1079.

25. Lüdtke A, et al. Hepatic steatosis in Dunnigan-type familial partial lipodystrophy. Am J Gastroenterol. 2005;100(10):2218-2224.

26. Misra A, Garg A. Clinical features and metabolic derangements in acquired generalized lipodystrophy: case reports and review of the literature. Medicine (Baltimore). 2003;82(2):129-146.

27. Bartelt A, et al. Brown adipose tissue activity controls triglyceride clearance. Nat Med. 2011;17(2):200-205.

28. Ouellet V, et al. Brown adipose tissue oxidative metabolism contributes to energy expenditure during acute cold exposure in humans. J Clin Invest. 2012;122(2):545-552.

29. Herman MA, et al. A novel ChREBP isoform in adipose tissue regulates systemic glucose metabolism. Nature. 2012;484(7394):333-338.

30. Samuel VT, Petersen KF, Shulman GI. Lipid-induced insulin resistance: unravelling the mechanism. Lancet. 2010;375(9733):2267-2277.

31. Yamauchi $\mathrm{T}$, et al. The fat-derived hormone adiponectin reverses insulin resistance associated with both lipoatrophy and obesity. 
Nat Med. 2001;7(8):941-946.

32. Peng XD, et al. Dwarfism, impaired skin development, skeletal muscle atrophy, delayed bone development, and impeded adipogenesis in mice lacking Akt1 and Akt2. Genes Dev. 2003;17(11):1352-1365.

33. Yun SJ, Kim EK, Tucker DF, Kim CD, Birnbaum MJ, Bae SS. Isoform-specific regulation of adipocyte differentiation by Akt/ protein kinase Balpha. Biochem Biophys Res Commun. 2008;371(1):138-143.

34. Bae SS, Cho H, Mu J, Birnbaum MJ. Isoform-specific regulation of insulin-dependent glucose uptake by Akt/protein kinase B J Biol Chem. 2003;278(49):49530-49536.

35. Baudry A, Yang ZZ, Hemmings BA. PKBalpha is required for adipose differentiation of mouse embryonic fibroblasts. J Cell Sci. 2006;119(Pt 5):889-897.

36. Nakae J, Kitamura T, Kitamura Y, Biggs WH, Arden KC, Accili D. The forkhead transcription factor Foxo1 regulates adipocyte differentiation. Dev Cell. 2003;4(1):119-129.

37. Kumar A, et al. Fat cell-specific ablation of rictor in mice impairs insulin-regulated fat cell and whole-body glucose and lipid metabolism. Diabetes. 2010;59(6):1397-1406.

38. Hung CM, et al. Rictor/mTORC2 loss in the Myf5 lineage reprograms brown fat metabolism and protects mice against obesity and metabolic disease. Cell Rep. 2014;8(1):256-271.

39. Ding Y, et al. Spemann organizer transcriptome induction by early beta-catenin, Wnt, Nodal, and Siamois signals in Xenopus laevis. Proc Natl Acad Sci U S A. 2017;114(15):E3081-E3090.

40. Kim MB, Song Y, Kim C, Hwang JK. Kirenol inhibits adipogenesis through activation of the Wnt/ $\beta$-catenin signaling pathway in 3T3-L1 adipocytes. Biochem Biophys Res Commun. 2014;445(2):433-438.

41. Xu H, Wang F, Wang J, Xu J, Wang Y, Xue C. The WNT/ $\beta$-catenin pathway is involved in the anti-adipogenic activity of cerebrosides from the sea cucumber Cucumaria frondosa. Food Funct. 2015;6(7):2396-2404.

42. Yu Y, et al. Kindlin 2 forms a transcriptional complex with $\beta$-catenin and TCF4 to enhance Wnt signalling. EMBO Rep. 2012;13(8):750-758.

43. Takada I, Kouzmenko AP, Kato S. Wnt and PPARgamma signaling in osteoblastogenesis and adipogenesis. Nat Rev Rheumatol. 2009;5(8):442-447.

44. Ducy P, et al. Leptin inhibits bone formation through a hypothalamic relay: a central control of bone mass. Cell. 2000;100(2):197-207.

45. Elefteriou F, et al. Serum leptin level is a regulator of bone mass. Proc Natl Acad Sci USA. 2004;101(9):3258-3263.

46. Kajimura D, et al. Adiponectin regulates bone mass via opposite central and peripheral mechanisms through FoxO1. Cell Metab. 2013;17(6):901-915

47. Zhang HQ, Wang LJ, Liu SH, Li J, Xiao LG, Yang GT. Adiponectin regulates bone mass in AIS osteopenia via RANKL/OPG and IL6 pathway. J Transl Med. 2019;17(1):64.

48. Wang T, He C, Yu X. Pro-inflammatory cytokines: New potential therapeutic targets for obesity-related bone disorders. Curr Drug Targets. 2017;18(14):1664-1675.

49. Gao H, et al. Suppression of CD36 attenuates adipogenesis with a reduction of P2X7 expression in 3T3-L1 cells. Biochem Biophys Res Commun. 2017;491(1):204-208.

50. Gao H, et al. Treatment with ginger ameliorates fructose-induced Fatty liver and hypertriglyceridemia in rats: modulation of the hepatic carbohydrate response element-binding protein-mediated pathway. Evid Based Complement Alternat Med. 2012;2012:570948.

51. Parlee SD, Lentz SI, Mori H, MacDougald OA. Quantifying size and number of adipocytes in adipose tissue. Meth Enzymol. 2014;537:93-122

52. Aune UL, Ruiz L, Kajimura S. Isolation and differentiation of stromal vascular cells to beige/brite cells. J Vis Exp. 2013;(73) doi: $10.3791 / 50191$

53. Bouxsein ML, Boyd SK, Christiansen BA, Guldberg RE, Jepsen KJ, Müller R. Guidelines for assessment of bone microstructure in rodents using micro-computed tomography. J Bone Miner Res. 2010;25(7):1468-1486. 